\title{
CONTINUOUS QUALITY IMPROVEMENT
}

\author{
MARY C. ZONSIUS AND KERRY A. MILNER
}

\section{THE HISTORY OF QUALITY IMPROVEMENT IN HEALTHCARE}

In the late 1980s, with the beginning transition from fee for service to a prospective payment plan, healthcare organizations were first challenged to evaluate their inefficiencies. With further changes in the financing of healthcare to rein in costs (e.g., capitation and contracting by large insurers and managed care organizations), hospitals began to recognize the competitive nature of healthcare and that inefficiency cut into profits. Healthcare organizations began to shift from quality assurance, a retrospective review of individual's compliance with policies and procedures, to proactive analysis of system's processes and outcomes called quality improvement (QI; Colton, 2000).

In addition to financial factors, several sentinel events accelerated the evolution of QI in healthcare organizations. The National Demonstration Project in QI in Health Care examined whether or not the industrial QI paradigm was transferable to healthcare organizations (Colton, 2000). The industrial QI paradigm was based on the founding work of Walter Shewhart, W. Edwards Deming, and Joseph Juran and results supported the use of this paradigm to drive patient safety and quality care in healthcare organizations (Burda, 1988). Bolstered by these results, co-author and physician Donald Berwick, made the case, in the New England Journal of Medicine, for using industrial-based QI methods to improve American healthcare (Berwick, 1989). Another sentinel event was The Joint Commission's Agenda for Change that included the use of QI in hospitals and included input from key stakeholders (e.g., patients, healthcare workers, and hospital administrators) to achieve its goal of rewriting its accreditation standards (Colton, 2000). The new standards focused on the QI process and included the use of run charts, Pareto charts and statistical process control to evaluate and improve systems to achieve better outcomes.

The Institute for Healthcare Improvement (IHI) emerged from the National Demonstration Project on QI in Health Care in 1991 in Cambridge, Massachusetts (IHI, n.d.). The creators of the IHI envisioned clinicians in healthcare systems using QI methods to fix unreliable common care practices (Berwick et al., 1990). The IHI recognized the need to build capacity for change in the U.S. healthcare system by creating an organization that would engage in knowledge sharing and training on performance improvement.

From 2005 to the present, the IHI has been establishing patient safety initiatives in the United States and abroad (IHI, n.d.). As part of this work, the IHI launched the Triple Aim in 2006 with a call to enhance the patient experience, improve population health, and reduce costs to optimize health system performance (Berwick et al., 2008). Clinicians in primary care practices across the United States have adopted the Triple Aim framework; however, stressful work life has interfered 
with their ability to achieve the three aims. In response to this, Bodenheimer and Sinsky (2014) proposed the Quadruple Aim that includes the tenets of the Triple Aim plus the aim of improving the work life of the healthcare workforce. The Quadruple Aim recognizes that a healthy workforce is needed to optimize health system performance.

\section{DEFINING CHARACTERISTICS OF QUALITY IMPROVEMENT IN HEALTHCARE}

The IHI, whose mission is to improve healthcare quality and safety for all, defines QI in terms of using a team of managers and staff that have the relevant expertise to analyze the current process (Scoville \& Little, 2014). This team is supported by QI specialists who work behind the scenes to improve the overall quality of care of that health facility. The team identifies the symptoms and causes of poor quality and devises a theory of what is needed to improve the process. The team uses a variety of improvement methods and tools to develop, test, and implement changes and redesigns the process if needed. The redesigned process is then monitored to ensure it performs at a new level (with new upper and lower control limits), with new work specifications, improved results, and reduced variation.

The National Quality Forum (NQF) was established in 1999 to improve the quality of U.S. healthcare. The forum works to define national goals and priorities for healthcare QI and develops standard metrics for measuring performance in healthcare nationally (Marjoua \& Bozic, 2012). They have metrics for processes (appropriate use), structures, outcomes, cost/resource use, and efficiency. The NQF stakeholders include hospitals, healthcare providers, consumer groups, purchasers, accrediting bodies, and research. Having national measures to assess healthcare performance is a critical component of ensuring appropriate and high-quality patient care.

The Health Resources and Services Administration (HRSA) is a federal agency whose mission is to improve access to healthcare and health outcomes of vulnerable populations. The HRSA defines QI as systematic and continuous actions that lead to measurable improvement in healthcare services and the health status of targeted patient groups (Department of Health and Human Services and HRSA, 2011). HRSA collaborates with other federal and state entities to improve care. For example, HRSA partners with the Centers for Medicare \& Medicaid Services (CMS) to improve care for vulnerable populations. The CMS defines QI as standardized behavior that is made systematic so that the same inputs result in the same outputs (CMS, n.d.). In this definition, behavior is aligned with best evidence. Donabedian's structure, process, and outcome (Donabedian, 2005) are used to increased desired health outcomes and Deming's PDSA (plando-study-act) cycle (Moen \& Norman, 2010) is used to standardize behavior.

The Quality and Safety Education for Nurses (QSEN) initiative began in 2005 with the mission of preparing competent nurses to improve the quality and safety of the healthcare system. The founding faculty members adapted the Institute of Medicine (IOM) competencies from the Health Professionals Education: A Bridge to Quality (Greiner \& Knebel, 2003) report and defined essential knowledge, skills, and attitudes (e.g., competency) in the areas of patient-centered care, evidence-based practice (EBP), teamwork and collaboration, safety, QI, and informatics for undergraduate (Cronenwett et al., 2007) and graduate nursing levels (American Association of Colleges of Nursing QSEN Education Consortium, 2012). The QSEN faculty defined QI in terms of "use data to monitor the outcomes of care processes and use improvement methods to design and test changes to continuously improve the quality and safety of healthcare systems" (Cronenwett et al., 2007, p. 127). QSEN has helped to bridge the gap between what is and what should be in delivering healthcare. 


\section{ADVANCED PRACTICE REGISTERED NURSE ENGAGEMENT IN QUALITY IMPROVEMENT}

The 2019 American Association of Nurse Practitioners (AANP) standards for practice include APRN engagement in QI. Standard VII: Quality Assurance and Continued Competence calls for "participation in quality assurance review, including the systematic, periodic review of records and plans of care that may result in quality improvement plan." Standard IX: Research as Basis for Practice calls for APRNs to "support research and dissemination of evidence-based practice by developing clinical research questions, conducting or participating in studies, implementing quality improvement, and incorporating system changes into practice" (AANP, n.d.).

Reimbursement is another key reason for APRNs to be engaged in QI activities. APRNs are eligible clinicians under the CMS value-based initiative called Merit-Based Incentive Payment System (MIPS; Curtin, 2019). In this new payment system, performance is measured using data that APRNs report in three areas: quality (50\% of score), promoting interoperability requirements (25\% of score) and improvement activities (15\% of score) and cost makes up the last $10 \%$. For quality, APRNs pick performance measures that best fit their practice from a provided list (e.g., appropriate use, patient safety, efficiency, patient experience or care coordination; AANP, n.d.). Promoting interoperability is fulfilled by hospitals and APRNs demonstrating compliance with information sharing. Improvement activities include how clinicians improve care processes, enhance patient engagement and access to care. It is assumed that these activities will promote ongoing improvement and innovation in healthcare.

Additionally, reimbursement under MIPS relies on performance measures. These performance measures should be based on metrics that assess the outcomes of APRN care on patient care and quality of care measures (Kapu \& Kleinpell, 2013). APRN outcomes must also be included in the benchmarks for hospital performance. Thus, APRNs must engage in the development of APRNassociated metrics in order to generate relevant data to accurately assess their performance and contributions to patient safety and quality care nationally.

\section{QUALITY IMPROVEMENT COMPETENCIES FOR ADVANCED PRACTICE REGISTERED NURSES}

The National Organization for Nurse Practitioner Faculty (NONPF) developed core competencies for nurse practitioners that include a quality competency. NONPF defines quality as the degree to which health services increase the desired health outcomes consistent with professional knowledge and standards, the understanding of how to access and use information databases, and how to critically evaluate research findings. Other QI competencies for nurse practitioners include:

- Uses best available evidence to continuously improve quality of clinical practice.

- Evaluates the relationships among access, cost, quality, and safety and their influence on healthcare.

- Evaluates how organizational structure, care processes, financing, marketing, and policy decisions impact the quality of healthcare.

- Applies skills in peer review to promote a culture of excellence.

- Anticipates variations in practice and is proactive in implementing interventions to ensure quality. 
TABLE 4.1 Comparison of Research, Evidence-Based Practice (EBP), Quality Improvement (Ql)

\begin{tabular}{|c|c|c|c|}
\hline & RESEARCH & EBP & Ql \\
\hline Definition & $\begin{array}{l}\text { Use scientific } \\
\text { method to } \\
\text { investigate a gap in } \\
\text { knowledge }\end{array}$ & $\begin{array}{l}\text { A problem-solving process that } \\
\text { integrates existing evidence } \\
\text { (research, Ql), nursing expertise } \\
\text { and patient preferences to } \\
\text { guide care decisions }\end{array}$ & $\begin{array}{l}\text { Appraise the efficiency } \\
\text { of clinical interventions } \\
\text { and provide guidance for } \\
\text { achieving quality outcomes, } \\
\text { productivity, cost containment }\end{array}$ \\
\hline Prompted by & Gap in knowledge & New evidence from research & $\begin{array}{l}\text { Process breakdown or system } \\
\text { failure }\end{array}$ \\
\hline Purpose & $\begin{array}{l}\text { Generate new } \\
\text { knowledge }\end{array}$ & $\begin{array}{l}\text { Integrate best evidence, } \\
\text { clinician's expertise, and } \\
\text { patient values and preferences } \\
\text { to improve health outcomes }\end{array}$ & $\begin{array}{l}\text { Improve system and process } \\
\text { of healthcare delivery }\end{array}$ \\
\hline Questions & $\begin{array}{l}\text { What is the best } \\
\text { thing to do? }\end{array}$ & Are we doing the best thing? & $\begin{array}{l}\text { Are we doing the best thing } \\
\text { right, all of the time? }\end{array}$ \\
\hline Results & $\begin{array}{l}\text { Generalizable to } \\
\text { population }\end{array}$ & $\begin{array}{l}\text { Recommendation for practice } \\
\text { change, clinical research study, } \\
\text { or no change }\end{array}$ & $\begin{array}{l}\text { Applicable to the patients } \\
\text { studied or local setting }\end{array}$ \\
\hline
\end{tabular}

\section{DIFFERENCES AMONG RESEARCH, EVIDENCE-BASED PRACTICE, AND QUALITY IMPROVEMENT}

APRNs need to be knowledgeable about the differences among research, EBP, and QI, and how they form the basis for practice inquiry. Table 4.1 outlines the specific characteristics of each method of inquiry. Research is pursued when there is a lack of knowledge upon which to base practice. EBP is a problem-solving approach to practice and EBP projects are undertaken when new evidence from research needs to be translated into practice. QI inquiry is followed when the current practice is the best thing to do (e.g., based on best available evidence), however there is breakdown in the process or structures, or the outcomes are not meeting expectations.

\section{QUALITY IMPROVEMENT AS THE CENTRAL COMPONENT OF THE NURSING INQUIRY PROCESS}

Figure 4.1 displays the Clinical Inquiry Process from Virginia Commonwealth University where performance improvement, also known as QI, is a central component of clinical inquiry. The EBP steps of problem identification, question development using the PICOT format, search for evidence, and appraisal of evidence appear across the top of Figure 4.1. If the Appraise Evidence step reveals adequate evidence but the current practice is not fully implemented, then the performance improvement (Process Enhancement/Improvement) inquiry should be followed. If the practice setting is not following the evidence-based recommendation for practice, then the EBP (Change in Practice) inquiry should be followed. If the current practice is fully implemented, then the APRN can stop or pursue new questions. If the Appraise Evidence step reveals a lack of evidence upon which to base practice, then the research inquiry (Creation of New Knowledge) should be followed. 


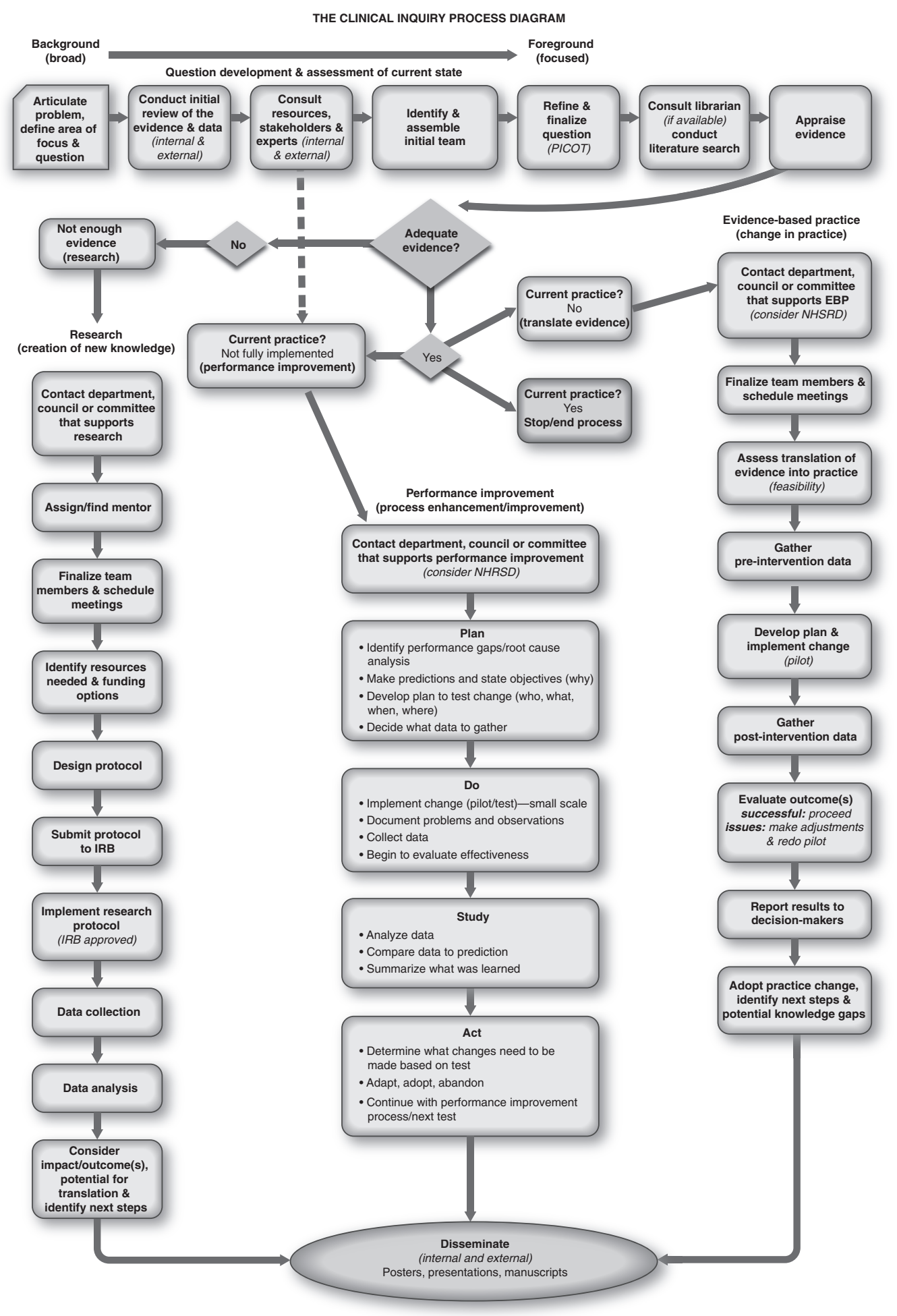

FIGURE 4.1 Nursing inquiry process diagram.

EBP, evidence-based practice; IRB, institutional review board; NHSRD, Not Human Subjects Research Determination; $\mathrm{PICOT}, \mathrm{P}=$ Patient population, $\mathrm{I}=$ Intervention, $\mathrm{C}=$ Comparison intervention, $\mathrm{O}=$ Outcome, $\mathrm{T}=$ Time.

Source: Used with permission from Roy, R. E. (2020). The Clinical Inquiry Process diagram. https:// scholarscompass.vcu.edu/libraries_pubs 


\section{THE QUALITY IMPROVEMENT PROCESS}

APRNs are well-suited to engage in the QI process because they have been prepared as critical thinkers and problem solvers in both their graduate education and in their foundational education (guided by the nursing process that is based on the scientific method). The QI process is considered an extension or rework of the scientific method (Cleghorn \& Headrick, 1996).

APRNs are integral to the QI process and armed with the knowledge and skills to navigate improvement at the micro, meso, and macro system levels. As a QI team leader, the APRN selects the QI model and tools that will improve the existing process. In practice there is not one "right" model to use and the choice may come down to the model with which the APRN and the QI team has the most experience (Silver et al., 2016). The APRN ensures the QI process is successful from start to finish and recognizes the importance of instilling a continuous improvement culture among the key stakeholders (Chandrasekaran \& Toussaint, 2019).

\section{QUALITY IMPROVEMENT MODELS}

This section provides an overview of five common QI models used by APRNs in the healthcare setting. Exemplars of how APRNS have used the QI models to improve practice are shared. Table 4.2 depicts how the steps of the nursing process closely align with the steps of each of the QI models. Moreover, the table shows that the models have more similarities than differences and have become more comprehensive across time.

\section{Donabedian Model of Care}

Donabedian was one of the early fathers of the quality movement who developed a model to evaluate the overall quality of medical care. The focus of the Donabedian Model of Care that was first

TABLE 4.2 Comparison of Common Quality Improvement Models

\begin{tabular}{|c|c|c|c|c|c|}
\hline \multirow[b]{2}{*}{$\begin{array}{l}\text { NURSING } \\
\text { PROCESS }\end{array}$} & \multicolumn{5}{|c|}{ COMMON QI MODELS } \\
\hline & $\begin{array}{l}\text { DONABEDIAN } \\
(1960 \mathrm{~s})\end{array}$ & $\begin{array}{l}\text { PDSA } \\
\text { (1920s) }\end{array}$ & $\begin{array}{l}\text { FOCUS- } \\
\text { PDCA } \\
\text { (1993) }\end{array}$ & $\begin{array}{l}\text { IHI MODEL FOR } \\
\text { IMPROVEMENT } \\
\text { (1990s) }\end{array}$ & $\begin{array}{l}\text { SIX SIGMA/ } \\
\text { DMAIC } \\
\text { (LATE 1980s) }\end{array}$ \\
\hline \multirow[t]{2}{*}{ Assess } & & & $\begin{array}{l}\text { Find/ } \\
\text { Organize }\end{array}$ & $\begin{array}{l}\text { What are we trying } \\
\text { to accomplish? }\end{array}$ & Define \\
\hline & & & Clarify & $\begin{array}{l}\text { How we know that } \\
\text { a change is an } \\
\text { improvement? }\end{array}$ & Measure \\
\hline Diagnose & & & $\begin{array}{l}\text { Understand/ } \\
\text { Select }\end{array}$ & $\begin{array}{l}\text { What change can we } \\
\text { make that will result } \\
\text { in an improvement? }\end{array}$ & Analyze \\
\hline Plan & Structure & Plan & Plan & Plan & \\
\hline Implement & Process & Do & Do & Do & Improve \\
\hline \multirow[t]{2}{*}{ Evaluate } & Outcome & Study & Check & Study & Control \\
\hline & & Act & Act & Act & \\
\hline
\end{tabular}


presented in the 1960s is an evaluation of quality that includes the triad of structure, process, and outcome (Donabedian, 2005). The thought was that to fully evaluate quality one had to have the proper structures in place that would influence the given process and ultimately the outcomes of care. Structure refers to the inputs; characteristically the setting where the process occurs, the staff involved in the process, and the material and organizational resources. Process is as stated, the process to be implemented and/or evaluated: What are the technical and interpersonal activities involved in delivering care? The outcome is the output, the effect of whether care of patients or population groups was improved (Donabedian, 1988).

The Donabedian model can be used to implement a QI change as well as to evaluate an existing process. APRNs Compton and Carrico (2018) used Donabedian's model to develop and implement a chronic obstructive pulmonary disease (COPD) tool to improve the patient and provider communication process and thereby improve patient outcomes by decreasing emergency department visits, hospitalizations, and healthcare costs.

\section{The Plan-Do-Study-Act (PDSA) Cycle}

The PDSA cycle that is commonly used in healthcare settings today stems from the original work of Walter Shewhart that began in the 1920s at Bell Telephone Laboratories that was later modified by W. Edwards Deming (Re \& Krousel-Wood, 1990). Together Shewhart and Deming are viewed as the founders of the industrial quality movement and fathers of the modern-day QI movement.

This four-step iterative model (PDSA) is best used to test interventions in a pilot setting using multiple cycles to refine the process prior to expanding system-wide. This model allows for rapid-cycle change (Taylor et al., 2014).

1. Plan. Assemble the team, understand current process, and identify possible solutions. Develop goals of the planned change and delineate who will do what and when to carry out the planned change.

2. Do. Implement the change and note any unexpected deviations from the plan.

3. Study. The analysis phase. Did the intervention go as planned? Was the plan successful? What was learned?

4. Act. If successful, sustain the change. If not, make modifications and retest.

The PDSA cycle is one of the most common QI models used by APRNs to improve the patient safety and quality care related to delirium (Fraire \& Whitehead, 2019), falls (Cangany et al., 2018; Grillo et al., 2019; Kohari, 2018), 30-day readmission rates (House et al., 2016), heparin infusion protocols (Johnson et al., 2018), pediatric asthma care (Kennedy \& Jolles, 2019), colon screening rates (Florea et al., 2016), sleep disturbances (Lopez et al., 2018), and medical management for older adults (Vejar et al., 2015).

\section{FOCUS-PDCA MODEL}

In 1993, the Hospital Corporation of America created the Find, Organize, Clarify, Understand, Select (FOCUS) model from Deming's PDSA model (Taylor et al., 2014) to guide the QI efforts of their healthcare workers. The five steps in this model are:

1. Find a process to improve.

2. Organize a team that knows the process.

3. Clarify current knowledge of the process. 
4. Understand causes of process variation.

5. Select the process improvement.

The five step FOCUS is followed by the Plan-Do-Check-Act (PDCA) cycle. However, small tests of change using PDCA are used in steps 3 and 4 to inform the improvement implemented in the PDCA cycle that begins following step 5 (Batalden \& Stoltz, 1993).

Note that in the PDCA cycle " $\mathrm{C}$ " is used for "Check" rather than the "S" for "Study." Many organizations use the "S" and "C" interchangeably although Deming stressed that semantically "check" was not appropriate because it means "to hold back" (Moen \& Norman, 2010).

The FOCUS-PDCA model provides additional structure and guidance to the planning of the QI process. An example of APRNs' use of this model in practice is the work by Watts and Nemes (2018) who used the FOCUS-PDCA model to guide the implementation of a hypoglycemic protocol to increase rechecks within 30 minutes of treatment.

\section{Institute for Healthcare Improvement Model for Improvement}

The IHI Model for improvement (MFI), an outgrowth of the more common PDSA cycle from Deming, was designed by Langley and colleagues concurrent to the FOCUS-PDCA model (Langley et al., 2009). The MFI, a comprehensive QI model, begins with three questions that inform the subsequent PDSA cycle. Note that the IHI MFI uses study for the third step of the cycle.

The three questions are (a) What are we trying to accomplish? - purpose or aim; (b) How do we know that a change is an improvement? - feedback/data are needed; and (c) What change can we make that will result in an improvement? - what specific change can be made that will address the purpose or aim? Next, rather than randomly implementing a change and hoping to get the expected result, the MFI supports a pilot test of the change using the PDSA model. The overall goal is to spread and sustain the change (Langley et al., 2009).

The IHI MFI provides additional structure and direction to guide the QI process. APRNs have used the model to improve suicide screening practices in an outpatient mental health clinic (Spear, 2018); implement a mobility plan in a long-term care facility (Kazana \& Murphy, 2018); and implement scripted post-discharge phone calls to heart failure patients to improve outcomes (Ruggiri et al., 2019).

\section{Six Sigma (DMAIC)}

The development of Six Sigma quality methodology, in the late 1980s, is attributed to the Motorola Corporation with the goal of decreasing process variability but also improving financial performance and customer satisfaction. The use of data to establish pre-change baseline performance, and statistical analysis tools distinguish this data-driven methodology from previous QI models that have been discussed (Glasgow et al., 2010; Takao, 2017).

Sigma uses the 5-step DMAIC model (define, measure, analyze, improve, and control):

1. Define. Identify the problem, scope of the project, key customer needs, and current process.

2. Measure. Collect data to determine the performance of the current process.

3. Analyze. Identify and prioritize root causes of variation.

4. Improve. Address opportunities for improvement in the process. Develop and implement solutions to reduce/eliminate root causes and decrease variation.

5. Control. Did the process improve? If so, sustain the gains. 
TABLE 4.3 DMAIC Tools

\begin{tabular}{|c|c|c|c|}
\hline & $\begin{array}{l}\text { SIX SIGMA } \\
\text { ANALYSIS TOOLS }\end{array}$ & $\begin{array}{l}\text { DESCRIPTION OF SIX SIGMA } \\
\text { TOOL }\end{array}$ & $\begin{array}{l}\text { COMMON QUALITY } \\
\text { IMPROVEMENT } \\
\text { TOOLS* }\end{array}$ \\
\hline Define & $\begin{array}{l}\text { Voice of the customer } \\
\text { (VoC) }\end{array}$ & $\begin{array}{l}\text { To gather customer feedback on needs/ } \\
\text { wants }\end{array}$ & Pareto diagram \\
\hline Measure & Value stream map & $\begin{array}{l}\text { Identify where in the process value is } \\
\text { enabled/added or nonvalue added. }\end{array}$ & \\
\hline Analyze & $\begin{array}{l}\text { Failure Effects Mode } \\
\text { Analysis (FMEA) }\end{array}$ & $\begin{array}{l}\text { Proactive assessment of the process to } \\
\text { identify where failure is occurring and } \\
\text { consequences of the failures. Prioritize } \\
\text { potential failures by severity/frequency. } \\
\text { Eliminate failures, addressing highest } \\
\text { priority first. }\end{array}$ & $\begin{array}{l}\text { Root cause analysis } \\
\text { Pareto diagram } \\
\text { Histogram } \\
\text { Brainstorming } \\
\text { Cause and effect - } \\
\text { Fishbone }\end{array}$ \\
\hline Improve & Kaizen events & $\begin{array}{l}\text { Rapid improvement event. Gathering of } \\
\text { key stakeholders to review and improve } \\
\text { process based on their knowledge of } \\
\text { the work. }\end{array}$ & \\
\hline Control & $\begin{array}{l}\text { Statistical process } \\
\text { control (SPC) e.g. } \\
\text { control charts }\end{array}$ & $\begin{array}{l}\text { Based on Shewhart's work - using } \\
\text { a statistical process to determine if } \\
\text { process variation is common cause or } \\
\text { special cause variation. }\end{array}$ & \\
\hline
\end{tabular}

*Common Ql tools are defined in the next section.

Additionally, Six Sigma analysis tools and common QI tools are used with each step of the DMAIC model. Extensive training is needed for effective use of the Six Sigma analysis tools described in Table 4.3. The common QI tools listed in the table are described later in the chapter (see Table 4.4).

The term Six Sigma is specific to manufacturing as it literally means to have less than 3.4 defects per 1 million opportunities. However, adaptations of Six Sigma have been used successfully to guide QI in the healthcare industry (Glasgow et al., 2010). APRNs have used the DMAIC model to successfully improve practice to decrease central line-associated bloodstream infection (CLABSI) rates below national benchmarks in a neurotrauma intensive care unit (Loftus et al., 2015).

TABLE 4.4 Common Quality Improvement Tools Categorized by Function

\begin{tabular}{|l|l|l|}
\hline $\begin{array}{l}\text { UNDERSTANDING THE } \\
\text { CURRENT PROCESS }\end{array}$ & IDENTIFYING ROOT CAUSE & DATA DISPLAY/ANALYSIS \\
\hline Process flowchart & Cause and effect/Fishbone & Histogram \\
\hline Benchmarking & 5 Whys & Pie chart \\
\hline Focus group & Pareto chart & Pareto chart \\
\hline & Focus groups & Run charts \\
\hline & & Control charts \\
\hline
\end{tabular}


Common QI models used by APRNs have been presented. It is interesting to note that Cleghorn and Headrick (1996) assert that the scientific method is (a) foundational to the education of health professionals and (b) that the QI process is an extension of or rework of the scientific method. This assertion supports why APRNs are well suited to engage in QI because both their foundational education (guided by the nursing process that is also based on the scientific method) and graduate education have prepared them as critical thinkers and problem solvers. Table 4.3 depicts how the steps of the nursing process closely align with the steps of each of the QI models. Moreover, the table shows that the models have more similarities than differences and have become more comprehensive across time.

\section{Quality Improvement Tools}

A graduate-level QSEN competency for QI is to "select and use quality improvement tools (e.g., run charts, control charts, root cause analysis, flow diagrams and GANTT charts) to achieve best possible outcomes" (American Association of Colleges of Nursing QSEN Education Consortium, 2012). Therefore, it is important that APRNs have a working knowledge of common QI tools. Common QI tools are used in tandem with the selected QI model to help achieve the desired result(s). As a rule, QI tools can be grouped by function: (a) understanding of the current process, (b) understanding of the root cause of the issue, and 3) data display/analysis (see Table 4.4).

\section{Quality Improvement Tools for Understanding the Current Process}

"If you do not ask the right questions, you do not get the right answers. A question asked in the right way often points to its own answer. Asking questions is the A-B-C of diagnosis. Only the inquiring mind solves problems."

-Edward Hodnett, poet (1841-1920)

One of the first steps in the QI process is to understand the current process, regardless of the QI model being followed. It is important to determine what is the baseline performance and what is working well before determining what can be improved. Commonly people rush to implement a solution before fully understanding the problem which leads to frustration and waste of resources. The process flowchart, benchmarking, and focus groups are three QI tools used to understand the current process.

\section{Process Flowchart}

The process flowchart or flow diagram is a graphic display of each step in a given process. Each step of the process is clarified and displayed from start to finish using a series of symbols connected by arrows to depict the chronological flow of the process. The rounded rectangle is used to depict the beginning and end of the process, rectangles denote steps in the process, and a diamond designates a decision (Heher \& Chen, 2017). See Figure 4.2.

Once the flowchart has been designed, a thoughtful analysis of the process helps to identify specific steps in the process where improvement opportunities exist (waste, duplication, complexity, delay). This analysis is best done with a team of committed stakeholders who can provide insight into the nuances of the process.

\section{Benchmarking}

Benchmarking is a tool used to evaluate if a process is meeting "best practices." The current metrics of a process are compared against the internal and/or external organizational standards to 


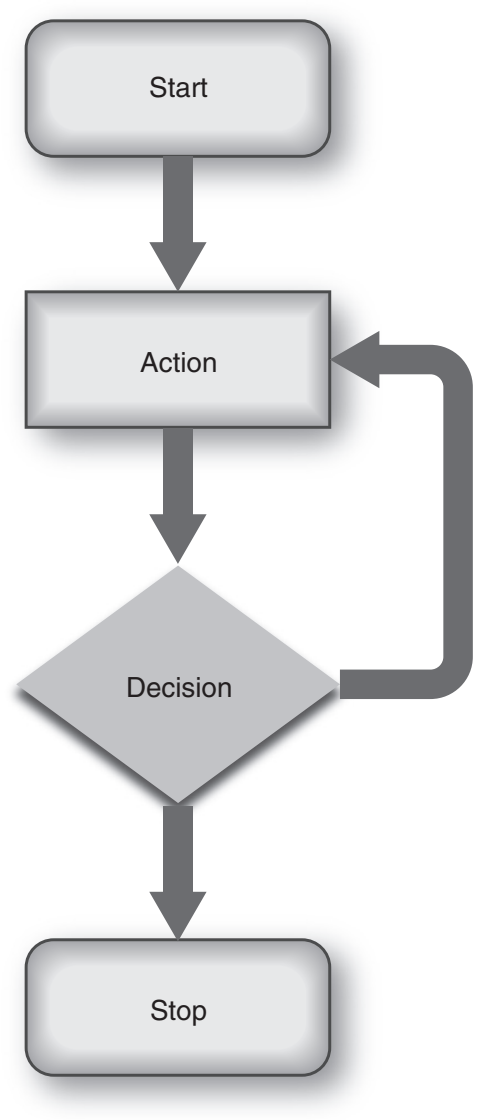

FIGURE 4.2 Example of a process flowchart.

provide baseline information on how well a process is currently performing (Wind \& van Harten, 2017). Sources for benchmarking include internal metrics that may be collected by the quality department in a health system. Performance and quality measures from national practice organizations (e.g. American Heart Association, American Diabetes Association, American College of Cardiology or the National Database of Nursing Quality Indicators) are sources of external benchmarks to evaluate quality of care.

A bar graph is one way to display the data to get a snapshot of current practice in relation to the benchmark against one or more data points. In Figure 4.3 the benchmark is the national $80^{\text {th }}$ percentile. In the first two time periods, the organization was below benchmark and above benchmark in the final three time periods.

\section{Focus Group}

A focus group is an effective strategy to gather baseline information from a group of stakeholders involved in a given process. This strategy can be used to identify stakeholder expectations as well as what they view as facilitators and barriers to the optimal performance of a process. Bringing small groups of stakeholders together face to face with a moderator allows for targeted and open conversation. Conducting a focus group takes careful planning and systematic delivery. Krueger and Casey (2015) support a step by step approach to conducting focus groups. 


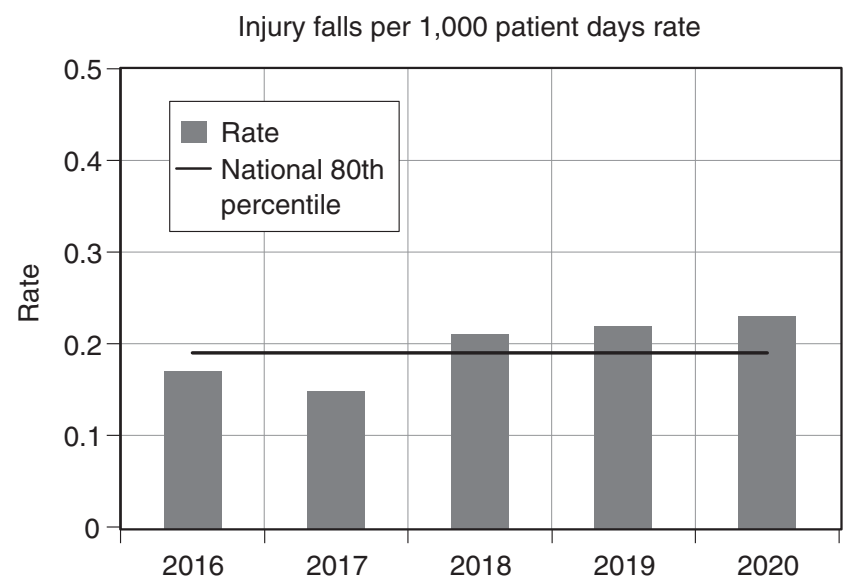

FIGURE 4.3 Benchmark with the use of a bar graph.

\section{Quality Improvement Tools for Understanding the Root Cause}

The cause-and-effect diagram (fishbone) and the 5 whys are two QI tools commonly used to help understand the root cause of a problem.

\section{Cause-and-Effect Diagram}

When attempting to establish the root cause of a problem A cause-and-effect diagram, also known as "Ishikawa" or "fishbone diagram, is commonly used to identify contributing factors impacting a problem. The problem (effect) is listed at the right of the diagram (the head) and the probably causes are placed on the diagonal lines (the bones). Typically, six categories are used to help sort the causes. As shown in Figure 4.4 these may include people (healthcare personnel), environment, process/ methods, patient. These categories can be altered as needed to guide the process. The best results are attained from a group brainstorming session to elicit multiple probable causes (Tague, 2005). Next the diagram is analyzed to review the cause-and-effect relationships among the probable causes.

\section{The 5 Whys}

Asking the " 5 whys" is a simple exercise to drill down to the ultimate root cause of a problem. To perform this exercise the learner repeatedly asks the question "why?" five times. The response to each "why" is one step closer to identifying the underlying cause of the problem. It is important that each "why" refocuses the user to keep digging deeper. The key to a successful " 5 whys" outcome is that the analysis must not end too early before the ultimate cause of the problem is identified. The process may take only two or three "whys" or could take more than five "whys" depending on the complexity of the problem (Barsalou, 2017). The " 5 whys" can also be used when performing a root cause analysis of a sentinel event or in combination with the fishbone as shown in Figure 4.4.

\section{Quality Improvement Tools for Data Analysis/Display}

"Data are not taken for museum purposes; they are taken as a basis for doing something. If nothing is to be done with the data, then there is no use in collecting any. The ultimate purpose of taking data is to provide a basis for action or a recommendation for action." 


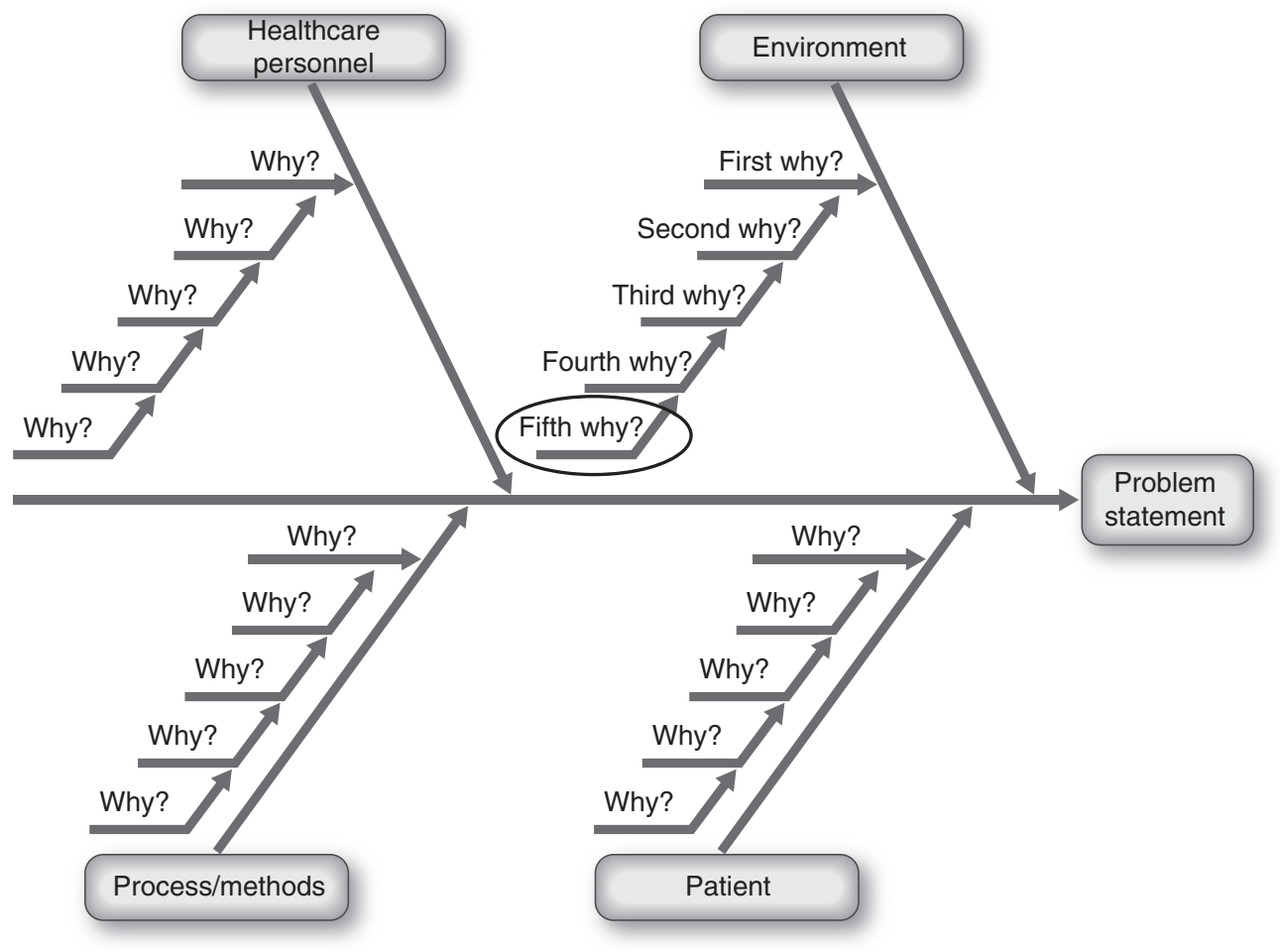

FIGURE 4.4 Fishbone diagram.

The histogram, bar chart, pie chart, and Pareto chart are common options to display frequency data (Dawson, 2019). First, consider whether the data are quantitative or categorical. The histogram is used for quantitative data whereas the bar, pie, and Pareto chart are used for categorical data. Displaying data by the frequency of occurrence provides a quick way to analyze visually the contributing factors to a problem.

\section{Histogram}

A histogram displays the frequency distribution of the values of a quantitative variable dataset (e.g., age, length of stay). This visual summary of the data can be used as an exploratory analysis tool to identify the range of the data, whether the data are normally distributed or skewed, and any outliers (Nuzzo, 2019). To construct a histogram, the horizontal or $x$ axis displays the quantitative variable and the frequency or count is graphed on the left vertical or $y$ axis and the percent on the right. Equally spaced adjacent columns are drawn to correspond with each of the quantitative variables and the height of each column aligns with the frequency. The highest column denotes the highest frequency. See Figure 4.5.

\section{Pareto Chart}

The Pareto chart is named for Vilfedo Pareto an Italian economist who in his study of income distribution identified the inequities of wealth distribution with a small percentage of the population holding the majority of the wealth (Pareto's law). Pareto's law is also known commonly as the $80 / 20$ rule. Juran transferred this principle to quality improvement, noting that a small percent of factors contributed to the most causes of defects (Kelly et al., 2013). Focusing on the $20 \%$ of 
Histogram: Falls per 1,000 patient days rate

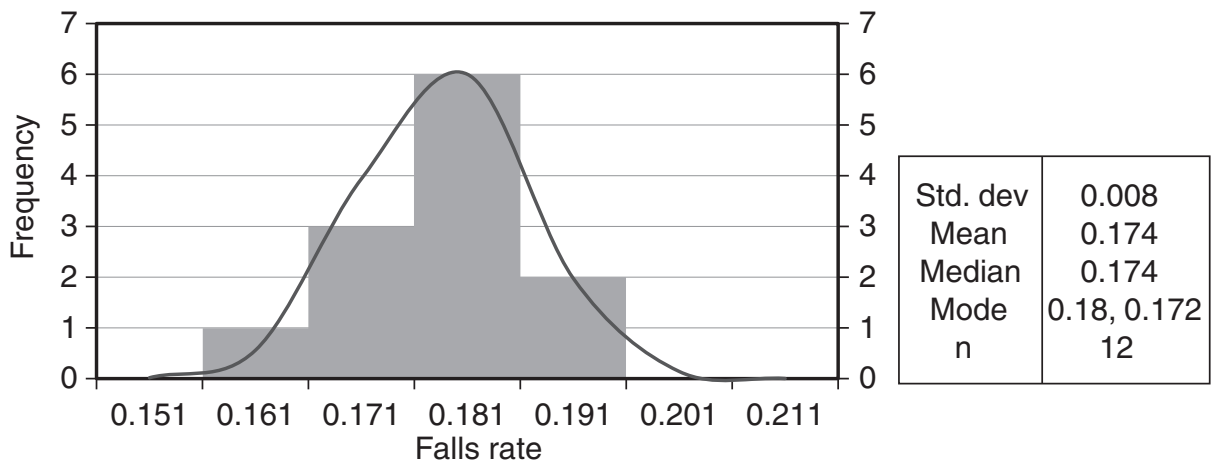

FIGURE 4.5 Example of a histogram.

causes that have the most direct impact on the effects or outcomes can improve process variation by $80 \%$. Simply stated, $80 \%$ of the problem can be resolved by addressing $20 \%$ of the root causes (Kelly et al., 2013; Livesay et al., 2020).

After identifying the contributing causes of variation, the Pareto chart (vertical bar graph) is used to determine which root causes are the most significant by displaying the most frequent causes left to right across the $\mathrm{x}$ axis. The categories of causes are depicted on the $x$ axis and the frequency is depicted by the $y$ axis (see Figure 4.6). The Pareto chart is best used for categorical data and provides a quick look at which contributing factor is occurring most frequently by displaying the descending order of frequency.

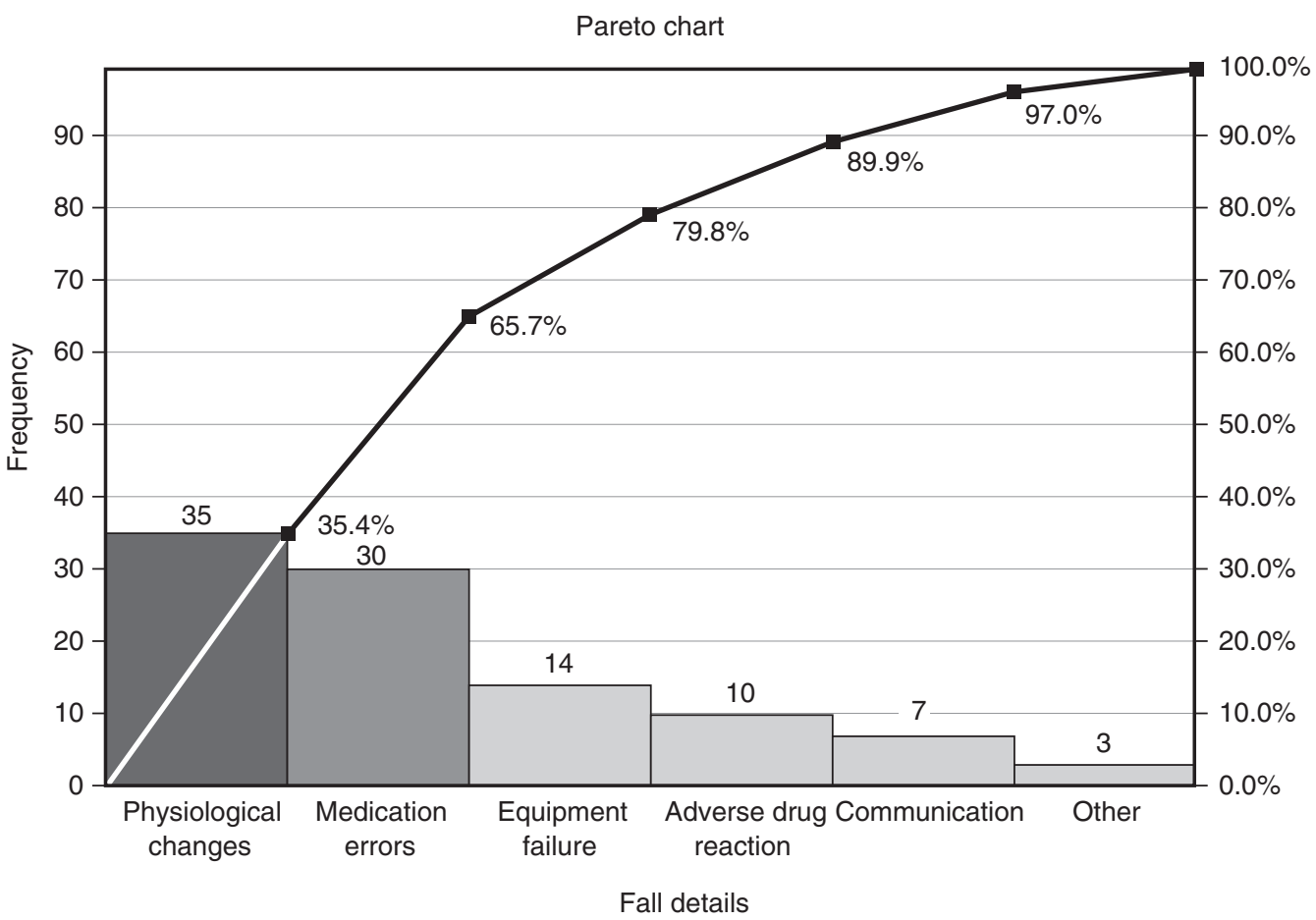

FIGURE 4.6 Example of a Pareto chart. 


\section{Bar Graph or Pie Chart}

A bar graph or pie chart can be used for categorical data such as gender or race (Nuzzo, 2016). The bar graph is displayed like the histogram, however there are gaps between the bars as each bar is self-contained whereas the histogram adds to $100 \%$. Relative frequency is displayed using a pie chart where each category is displayed as the percentage of the total (100\%) and the highest percentage is shown as the "biggest piece of the pie."

\section{Patterns/Trends in a Process}

Monitoring the QI process to observe patterns or trends in data across time is an important step to determine whether an improvement has occurred. Generally, 15 or more data points are needed to strengthen the data interpretation (Lloyd, 2019). Two tools to display the data include a run chart and a control chart. The control chart, also known as the Shewhart chart or statistical process control chart (SPC), was developed by Shewhart during his work at Bell Laboratories in the 1920s (Connelly, 2018).

\section{Run Chart}

The run chart or timed-series chart displays data across time using the $x$ axis as the time scale and the $y$ axis as the frequency measure in number, percent, or rate (e.g., the fall rate per month). The distinctive feature of a run chart is a line drawn at the median where half of the data points fall above, and half the data points fall below. The run chart provides a visual and analytical display of the process performance and can be used to detect process variation after the implementation of a planned change (Perla et al., 2011). It allows for a quick display of the progress of a planned change to determine if the planned intervention is having the intended effect and whether that effect is sustained (Provost \& Murray, 2011).

To interpret the data on a run chart, there are two basic rules that can be used "to identify nonrandom signals on a run chart" (Lloyd, 2019, p. 192). The first step is to review how the data points cluster either above or below the median. A shift occurs when there are six or more consecutive data points clustered either above or below the median. Data points directly on the median are not counted. A trend is considered when there are five or more consecutive points that are moving in the same direction - either all up or all down (Lloyd, 2019; Perla et al., 2011).

\section{Control Chart (Also Known as the Shewhart Chart or Statistical Process Control Chart)}

Like a run chart, a control chart provides a visual display of process variation and is one tool to monitor and evaluate the effect of the planned change/QI across time. However, the control chart provides a more detailed approach to determine whether the process variations is normal fluctuation, in control (within the control limits), or is out of control (special cause variation; Fretheim \& Tomic, 2015). Ideally, adequate baseline data are also plotted so one can review the process variation pre-implementation of the planned change (Shaughnessy et al., 2018).

For a control chart the $x$ and $y$ axes are constructed similarly to the run chart, however a minimum of 20 data points is suggested for the control chart (Lloyd, 2019). Between the two charts, the key differences are that the mean is used for the central line, and upper control limits (UCL) and lower control limits (LCL) are calculated and inserted on the graph as an upper and lower line. Often the UCL and the LCL are described as three standard deviations from the mean, however the IHI and others stress that this is inaccurate and technically the upper and lower limits should reflect 


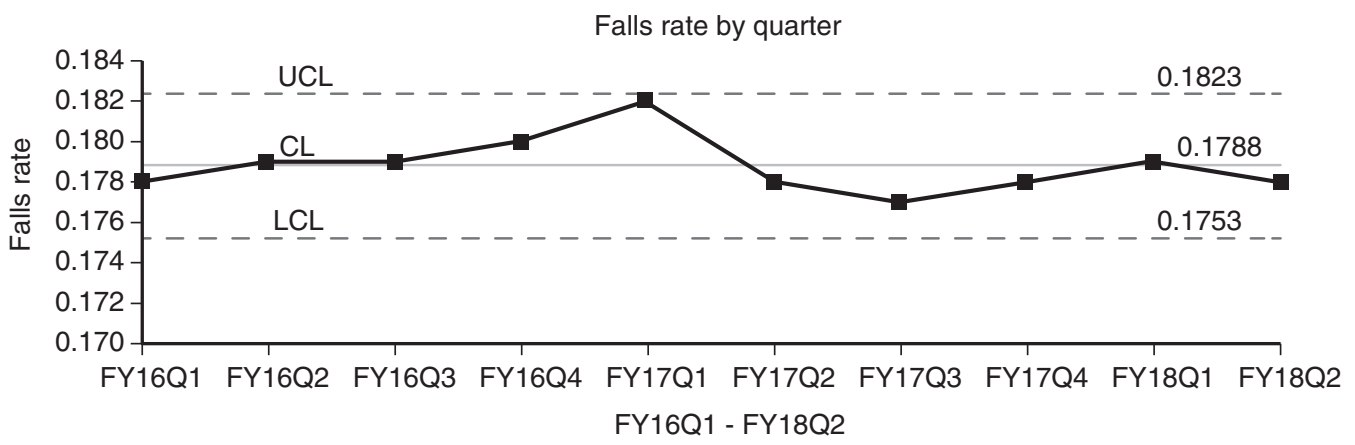

FIGURE 4.7 A control chart with common-cause variation.

$\mathrm{CL}$, central line; $\mathrm{LCL}$, lower control limit; $\mathrm{UCL}$; upper control limit.

$+/-3$ sigma limits from the mean (Lloyd, 2019). The limits can be determined readily using a software program such as Microsoft Excel. Remember to mark on the chart when the planned change or other contributing events occurred as this information strengthens the data interpretation.

\section{Within the Control Limit}

When reviewing the control chart, two types of process variation are considered, common-cause and special-cause variation. A common-cause variation (Figure 4.7) points to expected process variation. In this case, the process variation is attributed to expected fluctuations and the process is considered to be "in control" (Brady et al., 2018; Fretheim \& Tomic, 2015). However, a special-cause variation (Figure 4.8) suggests that a change in the process has occurred due to a specific cause, most likely the planned intervention has affected a true change in the process.

The IHI outlines five control chart analysis rules that can be used to evaluate the data and detect special causes, three of which are outlined here (Lloyd, 2019). Rule 1 is one data point that is outside either the UCL or LCL and is named a 3-sigma violation. Given that this is an outlier, the first step to take is to verify the origin of the data point. Rule 2 refers to a shift, visualized by eight consecutive points either above or below the mean. This differs from the

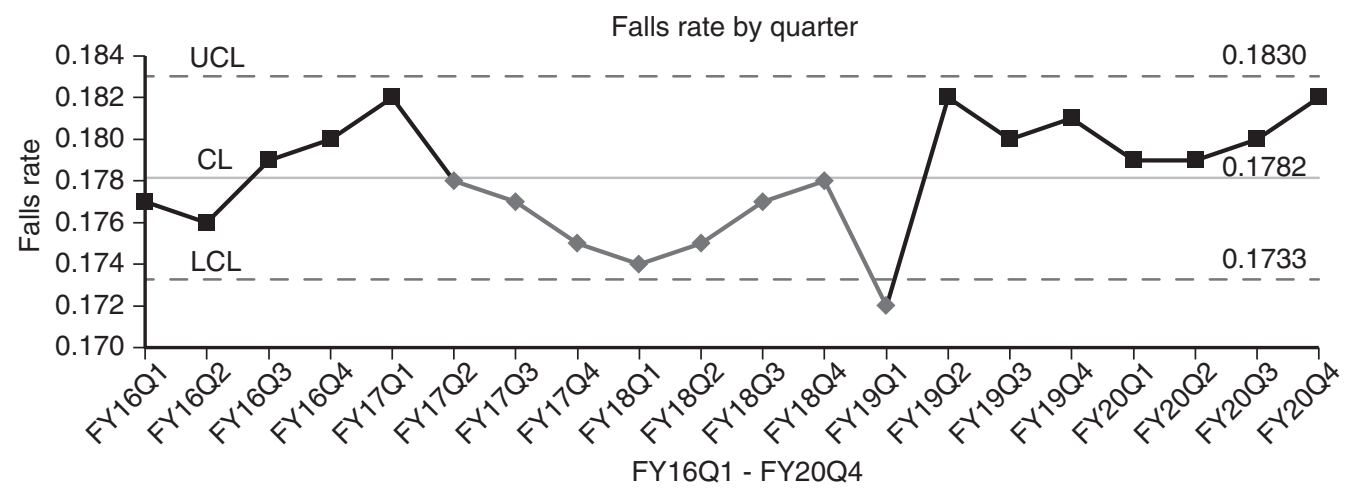

FIGURE 4.8 Special-cause variation, Rule 2 - a shift, eight consecutive points below the mean, one data point outside the lower control limits.

$\mathrm{CL}$, central line; LCL, lower control limit; UCL; upper control limit. 


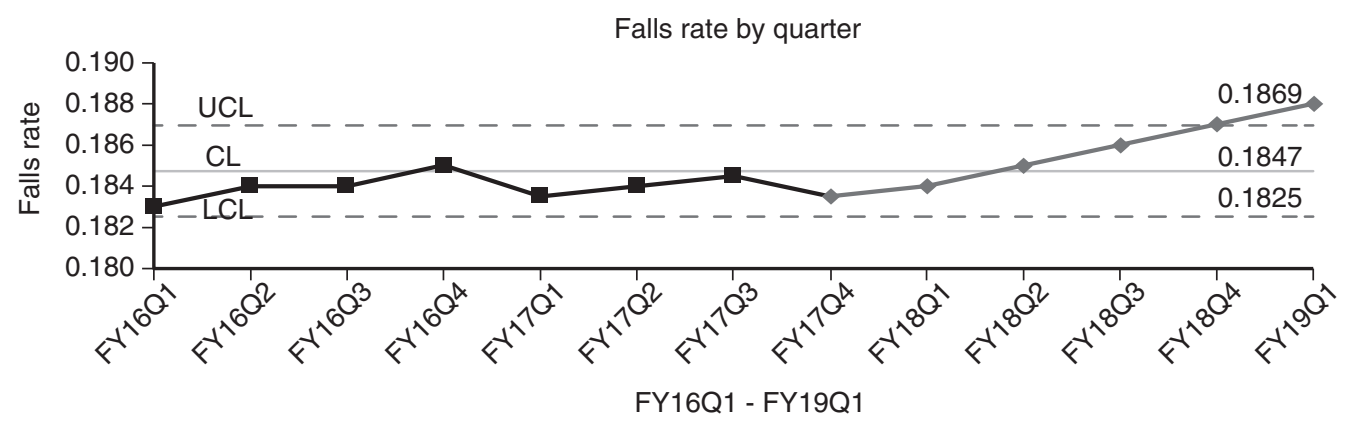

FIGURE 4.9 Special-cause variation, Rule 3 - a trend, six data points moving consecutively upward, one data point outside the upper control limits.

$\mathrm{CL}$, central line; $\mathrm{LCL}$, lower control limit; UCL; upper control limit.

shift on a run chart where only six consecutive points are needed. A shift-provided it is in the desired direction -is an indication that the proposed change is having the desired effect across time (see Figure 4.8). Rule 3 is commonly known as a trend and consists of minimally six data points that are moving consecutively either upward or downward. Similar to a shift, the trend is a favorable outcome and can indicate that true change is occurring within the process (see Figure 4.9) (Lloyd, 2019).

It is important to remember that all change visually depicted on a control chart is not special-cause variation; therefore, not every change in a process should be responded to or celebrated. Often in healthcare, leaders are quick to react to a downward or upward turn of one or two data points without having sufficient data to make an effective interpretation. The importance of the role of advance practice nurse leaders who can accurately interpret the data and contribute to these conversations can't be emphasized enough.

\section{SUMMARY}

The APRN is in a pivotal role to direct QI in the practice setting. APRNs are equipped with the QI knowledge and skills to support quality care and patient safety in health systems. The common QI model/processes and tool used by APRNs to develop and lead QI project plans are reviewed in this chapter.

\section{SUGGESTED LEARNING ACTIVITY}

1. Complete the low-tech simulation exercise "Mr. Potato Head: A LEAN, Mean Quality Improvement Teaching Machine!" found on the QSEN website at https://qsen.org/ This exercise addresses each of the QSEN graduate RN competencies and ideally is completed in teams of seven to eight members.

2. The IHI website houses many valuable resources to assist with learning the QI process. Two specific resources to be aware of are the IHI's QI Essentials Toolkit, which provides templates for each of the common QI tools listed in Table 4.4, and The Science of Improvement on a Whiteboard! that features a collection of 20 short videos covering topics from Deming to control charts. Available on-line at www.ihi.org/ 


\section{REFERENCES}

American Association of Colleges of Nursing QSEN Education Consortium. (2012). Graduate-Level QSEN Competencies: Knowledge, Skills, and Attitudes. American Association of Colleges of Nursing. https://www.aacnnursing.org/Portals/42/AcademicNursing/CurriculumGuidelines/Graduate-QSEN -Competencies.pdf

American Association of Nurse Practitioners. (n.d.). Standards of practice for nurse practitioners. https:// www.aanp.org/advocacy/advocacy-resource/position-statements/standards-of-practice-for-nurse -practitioners

Barsalou, M. (2017). Square in the crosshairs. Quality Progress, 50(1), 24-28.

Batalden, P., \& Stoltz, P. (1993). A framework for the continual improvement of health care: Build and applying professional and improvement knowledge to test changes in daily work. The Joint Commission Journal of Quality Improvement, 19, 424-447. https://doi.org/10.1016/S1070-3241(16)30025-6

Berwick, D. M. (1989). Continuous improvement as an ideal in health care. The New England Journal of Medicine, 320(1), 53-56. https://doi.org/10.1056/NEJM198901053200110

Berwick, D. M., Godfrey, A. B., \& Roessner, J. (1990). Curing health care: New strategies for suality improvement. Jossey-Bass.

Berwick, D. M., Nolan, T. W., \& Whittington, J. (2008). The triple aim: Care, health, and cost. Health Affairs (Project Hope), 27(3), 759-769. https://doi.org/10.1377/hlthaff.27.3.759

Bodenheimer, T., \& Sinsky, C. (2014). From triple to quadruple aim: Care of the patient requires care of the provider. Annals of Family Medicine, 12(6), 573-576. https://doi.org/10.1370/afm.1713

Brady, P. W., Tchou, M. J., Ambroggio, L., Schondelmeyer, A. C., \& Shaughnessy, E. E. (2018). Quality improvement feature series article 2: Displaying and analyzing quality improvement data. Journal of Pediatric Infectious Disease Society, 7(2), 100-103. https://doi.org/10.1093/jpids/pix077

Burda, D. (1988). Providers look to industry for quality models. Modern Healthcare, 18(29), 24-26, 28, 30-32.

Cangany, M., Peters, L., Gregg, K., Welsh, T., \& Jimison, B. (2018). Preventing falls: Is no toileting alone the answer? MEDSURG Nursing, 27(6), 379-382.

Centers for Medicare \& Medicaid Services. (n.d.). Quality Measure and Quality Improvement. https:// www.cms.gov/Medicare/Quality-Initiatives-Patient-Assessment-Instruments/MMS/Quality-Measure -and-Quality-Improvement-

Chandrasekaran, A., \& Toussaint, J. S. (2019, May 24). Creating a culture of continuous improvement. Harvard Business Review. https://hbr.org/2019/05/creating-a-culture-of-continuous-improvement? referral $=03758 \& \mathrm{~cm} \_v c=r r \_$item_page.top_right

Cleghorn, G. D., \& Headrick, L. A. (1996). The PDSA cycle at the core of learning in health professions education. Joint Commission Journal on Quality Improvement, 22(3), 206-212. https://doi.org/10.1016/ s1070-3241(16)30223-1

Colton, D. (2000). Quality improvement in health care. Evaluation \& the Health Professions, 23(1), 7-42. https://doi.org/10.1177/01632780022034462

Compton, J. J., \& Carrico, C. (2018). Development and implementation of an after-visit summary for COPD patients: A pilot QI project. Unpublished manuscript, College of Nursing, Creighton University, Omaha, Nebraska, US.

Connelly, L. (2018). Statistical process control. Medsurg Nursing, 27(5), 331-332.

Cronenwett, L., Sherwood, G., Barnsteiner, J., Disch, J., Johnson, J., Mitchell, P., Sullivan, D. T., \& Warren, J. (2007). Quality and safety education for nurses. Nursing Outlook, 55(3), 122-131. https://doi .org/10.1016/j.outlook.2007.02.006

Curtin, L. (2019). What you need to know about MIPS. American Nurse Today, 14(3), 48.

Dawson, A. (2019). A practical guide to performance improvement: Data collection and analysis. AORN Journal. 109(5), 621-631. https://doi.org/10.1002/aorn.12673

Donabedian, A. (1988). The quality of care. How can it be assessed? Journal of the American Medical Association, 260(12), 1743-1748. https://doi.org/10.1001/jama.1988.03410120089033

Donabedian, A. (2005). Evaluating the quality of medical care. The Milbank Quarterly, 83(4), 691-729. https://doi.org/10.1111/j.1468-0009.2005.00397.x

Florea, K. S., Novosel, L. M., \& Schlenk, E. A. (2016). Improvement in colon cancer screening through use of a multilevel intervention: A QI initiative. Journal of the American Association of Nurse Practitioners 28, 362-369. https://doi.org/10.1002/2327-6924.12320 
Fraire, M. L., \& Whitehead, D. K. (2019). Reducing delirium in hospitalized older adults with a nursing prevention protocol. MEDSURG Nursing, 28(2), 114-118.

Fretheim, A., \& Tomic, O. (2015). Statistical process control and interrupted time series: A golden opportunity for impact evaluation in quality improvement. BMJ Quality \& Safety, 24, 748-752. https://doi .org/10.1136/bmjqs-2014-003756

Glasgow, J. M., Scott-Caziewell, J. R., \& Kaboli, P. J. (2010). Guiding inpatient quality improvement: A systematic review of Lean and Six Sigma. The Joint Commission Journal on Quality and Patient Safety, 36(12), 533-540. https://doi.org/10.1016/s1553-7250(10)36081-8

Greiner, A. C., \& Knebel, E. (2003). Health professions education: A bridge to quality. National Academies Press.

Grillo, D. M., Firth, K. H., \& Hatchel, K. (2019). Implementation of purposeful hourly rounds in addition to a fall bundle to prevent inpatient falls on a medical-surgical acute hospital unit. MEDSURG Nursing, 28(4), 243-246, 261.

Heher, Y. K. \& Chen. Y. (2017). Process mapping: A cornerstone of quality improvement. Cancer Cytopathology, 125(12), 887-890. https://doi.org/10.1002/cncy.21946

House, M., Stephens, K. P., Whiteman, K., Swanson-Biearman, B., \& Printz, M. (2016). Cardiac medicine 30-day readmission reduction strategies: Do improved discharge transitions decrease readmissions? MEDSURG Nursing, 25(4), 251-254.

Institute for Healthcare Improvement. (n.d.). The IHI timeline: More than 25 years of driving improvement. http://www.ihi.org/about/Documents/IHI_Timeline_2019.pdf

Johnson, C., Miltner, R., \& Wilson, M. (2018). Increasing nurse-driven heparin infusion administration safety: A quality improvement initiative. MEDSURG Nursing, 27(4), 243-246.

Kapu, A. N., \& Kleinpell, R. (2013). Developing nurse practitioner associated metrics for outcomes assessment. Journal of the American Association of Nurse Practitioners, 25(6), 289-296. https://doi .org/10.1111/1745-7599.12001

Kazana, I., \& Murphy, M. P. (2018). Implementing a patient-centered walking program for residents in longterm care: A quality improvement project. Journal of the American Association of Nurse Practitioners, 30(7), 383-391. https://doi.org/10.1097/JXX.0000000000000037

Kelly, D. L., Johnson, S. P., \& Sollecito, W. A. (2013). Measurement, variation, and CQI tools. In W. A. Sollecito \& J. K. Johnson (Eds.), McLaughlin and Kaluzny's continuous quality improvement in health care (4th ed., pp. 77-114). Jones \& Bartlett.

Kennedy, C. M., \& Jolles, D. R. (2019, November). Providing effective asthma care at a pediatric patient-centered medical home. Journal of the American Association of Nurse Practitioners. Advance online publication. https://doi.org/10.1097/JXX.0000000000000334

Kohari, A. N. (2018). Improving fall risk assessment and documentation: A QI project. University of Kentucky DNP Projects. 195. https://uknowledge.uky.edu/dnp_etds/195

Krueger, R. A., \& Casey, M. A. (2015). Focus Groups: A Practical Guide for Applied Research (5th ed.). SAGE.

Langley, G. J., Moen, R. D., Nolan, K.M., Nolan, T. W., Norman, C. L., \& Provost, L. P. (2009). The improvement guide: A practical approach to enhancing organizational performance (2nd ed.). Jossey-Bass.

Livesay, S., Zonsius, M., \& McNett, M. (2020). Evaluating data to guide care delivery: Quality improvement methods and implementation science. In M. McNett (Ed.), Data for nurses (pp. 59-84). Elsevier.

Lloyd, R. C. (2019). Quality health care: A guide to developing and using ndicators (2nd ed.). Jones \& Bartlett.

Loftus, K., Tilley, T., Hoffman, J., Bradburn, E., \& Harvey, E. (2015). Use of six sigma strategies to pull the line on central line-associated bloodstream infections in a neurotrauma intensive care unit. Journal of Trauma Nursing, 22(2), 78-86. https://doi.org/10.1097/JTN.0000000000000111

Lopez, M., Blackburn, L., \& Springer, C. (2018). Minimizing sleep disturbances to improve patient outcomes. MEDSURG Nursing, 27(6), 368-371.

Marjoua, Y., \& Bozic, K. J. (2012). Brief history of quality movement in US healthcare. Current Reviews in Musculoskeletal Medicine, 5(4), 265-273. https://doi.org/10.1007/s12178-012-9137-8

Moen, R., \& Norman, C. (2010). Circling back. Clearing up the myths regarding the Deming cycle and seeing how it keeps evolving. Quality Progress, 43(11), 22-28.

Nuzzo, R. L. (2016). Statistically speaking. The box plots alternative for visualizing quantitative data. Physical Medicine and Rehabilitation Journal, 8(3), 268-272. https://doi.org/10.1016/j.pmrj.2016.02.001

Nuzzo, R. (2019). Histograms: A useful data analysis visualization. American Academy of Physical Medicine and Rehabilitation, 11(3), 309-312. https://doi.org/10.1002/pmrj.12145 
Perla, R. J., Provost, L. P., \& Murray, S. K. (2011). The run chart: A simple analytical tool for learning from variation in healthcare processes. BMJ Quality \& Safety, 20, 46-51. https://doi.org/10.1136/bmjqs.2009.037895

Provost, L. P., \& Murray, S. (2011). The Health Care Data Guide: Learning from Data for Improvement. Jossey-Bass.

Re, R. N., \& Krousel-Wood, M. A. (1990). How to use continuous quality improvement theory and statistical control tools in a multispecialty clinic. Quality Review Bulletin, 16(11), 391-397. https://doi.org/10.1016/ s0097-5990(16)30398-0

Roy, R. E. (2018). VCU Health Nursing inquiry process diagram (version 2). https://scholarscompass.vcu.edu/ libraries_pubs/52

Ruggiri, J. C., Milner, K. A., \& Buonocore, D. (2019). Implementing post-discharge 48- hour scripted call for patients with heart failure: An evidence-based practice quality improvement project. MEDSURG Nursing, 28(3), 183-187.

Scoville, R., \& Little, K. (2014). Comparing Lean and Quality Improvement. IHI White Paper.

Shaughnessy, E. E., Shah, A., Ambrogio, L., \& Statile, A. (2018). Quality improvement feature series article1: Introduction to quality improvement. Journal of Pediatric Infectious Disease Society, 7(1), 6-10. https:// doi.org/10.1093/jpids/pix061

Silver, S. A., Harel, Z., McQuillan, R., Weizman, A. V., Thomas, A. Chertow, G. M., Nesrallah, G., \& Chan, C. T. (2016). How to begin a quality improvement project. Clinical Journal of the American Society of Nephrology, 11, 893-900. https://doi.org/10.2215/CJN.11491015

Spear, V. (2018). DNP student quality improvement proposal: Implementing suicide protocol in outpatient mental health clinic. Scholar Archive, 4043. https://digitalcommons.ohsu.edu/etd/4043

Tague, N. R. (2005). The quality toolbox (2nd ed.). ASQ Quality Press.

Takao, M. R. V., Woldt, J., \& Bento da Silva, I. (2017). Six Sigma methodology advantages for small- and medium-sized enterprises: A case study in the plumbing industry in the United States. Advances in Mechanical Engineering, 9(10), 1-10. https://doi.org/10.1177/1687814017733248

Taylor, M. J., McNicholas C., Nicolay C., Darzi A., Bell, D., \& Reed J. E. (2014). Systematic review of the application of the Plan-Do-Study-Act method to improve quality in healthcare. BMJ Quality \& Safety, 23(4), 290-298. https://doi.org/10.1136/bmjqs-2013-001862

U.S. Department of Health and Human Services, \& Health Resources and Services Adminstration. (2011). Quality Improvement. https://www.hrsa.gov/sites/default/files/quality/toolbox/508pdfs/ qualityimprovement.pdf

Vejar, M. V., Makic, M. B. F., \& Kotthoff-Burnell, E. (2015). Medication management for elderly patients in an academic primary care setting: A quality improvement project. Journal of the American Association of Nurse Practitioners, 27(2), 72-78. https://doi.org/10.1002/2327-6924.12121

Watts, S. A., \& Nemes, D. (2018). Best practice nursing management of nosocomial hypoglycemia: Lessons learned. MEDSURG Nursing, 27(2), 98-102.

Wind, A., \& van Harten, W. H. (2017). Benchmarking specialty hospitals, a scoping review on theory and practice. BMC Health Services Research, 17(1), 245. https://doi.org/10.1186/s12913-017-2154-y 